\title{
Combing Location-Aware Applications with in-situ Actors Performances
}

\author{
Federico M. Alconada Verzini \\ LIFIA, Fac. de Informática, UNLP. \\ Calle 50 y 120, La Plata, \\ Buenos Aires, Argentina. \\ +54 $221422-8252$ \\ falconada @lifia.info.unlp.edu.ar
}

\author{
Cecilia Challiol $^{\ddagger}$ \\ LIFIA, Fac. de Informática, UNLP. \\ Calle 50 y 120, La Plata, \\ Buenos Aires, Argentina. \\ +54 $221422-8252$ \\ ceciliac@lifia.info.unlp.edu.ar
}

\author{
Alejandra B. Lliteras \\ LIFIA, Fac. de Informática, UNLP. \\ Calle 50 y 120, La Plata, \\ Buenos Aires, Argentina. \\ +54 221 422-8252 \\ Iliteras@lifia.info.unlp.edu.ar
}

\author{
Silvia E. Gordillo ${ }^{\S}$ \\ LIFIA, Fac. de Informática, UNLP. \\ Calle 50 y 120, La Plata, \\ Buenos Aires, Argentina. \\ +54 221 422-8252 \\ gordillo@lifia.info.unlp.edu.ar
}

\begin{abstract}
In this paper we present a model to define location-aware experiences having its different aspects (contents, location, etc) decoupled one from another. We have created an application following this model, which is also combined with actors' performances. This application defines three relevant locations inside a specific building. By reading a QR-code the user indicates that he/she has arrived at one of those locations. In that moment, the application triggers some questions related to the actors' performances. Hence, the user is required to see all the plays in order to be able to answer such questions. The application assists the user in moving throughout the different locations. We present the evaluation of this application and then we discuss interesting aspects that have shown up as a result of the in-situ evaluation of this application.
\end{abstract}

\section{Categories and Subject Descriptors}

D.2.13 [Software Engineering]: Reusable Software - Reuse models

H.5.2 [Information Interfaces and Presentation]: User Interfaces - Prototyping

\section{General Terms: Design, Experimentation}

Keywords: Location-Aware Applications, In-situ Actors Performance, In-situ evaluation, Prototype, Modelling LocationAware Applications, Separation of Concerns, Mobile Computing

\section{INTRODUCTION}

Mobile Applications have been increasing in the last few years covering different domains such as education, entertainment,

\footnotetext{
Permission to make digital or hard copies of all or part of this work for personal or classroom use is granted without fee provided that copies are not made or distributed for profit or commercial advantage and that copies bear this notice and the full citation on the first page. Copyrights for components of this work owned by others than ACM must be honored. Abstracting with credit is permitted. To copy otherwise, or republish, to post on servers or to redistribute to lists, requires prior specific permission and/or a fee. Request permissions from Permissions@acm.org.

NHT'15, September 1, 2015, Guzelyurt, Northern Cyprus. (C) 2015 ACM. ISBN 978-1-4503-3797-7/15/09 ..\$15.00DOI:

http://dx.doi.org/10.1145/2804565.2804571
}

tourist guides, etc. In particular, those applications that reference some relevant aspects of the environment (e.g. location-aware application) have only been tested in an in-situ fashion. Different authors ([2], [3], [14] and [16]) have come to the conclusion that evaluating prototypes in-situ is essential. In [12], the authors reinforce this idea by explaining that the way in which a person perceives a physical space can change according to the time of the day (morning, afternoon, evening), for instance.

There are different ways of creating mobile applications. In particular, location-aware applications can be defined by developers (e.g. [8], [10]) or by end-user ([13] and [15]). In both cases, according to what was mentioned before, the final mobile application is required to be tested in-situ to obtain real evaluation results.

Mobile Urban Dramas are a particular type of mobile application introduced in [7] and defined as Location-Aware Narratives. In each location defined within a narrative, the user receives a multimedia content, such as an audio or an image, and then he/she continues to the next location. Recent works of these authors present six different dramas [8], which are defined with specific domains (e.g. education or entertainment). Inspired by these dramas we came up with the idea of creating a location-aware application combined with plays performed by real actors. For each relevant location, the application shows a specific content and several actors perform a play. The same play can be performed in different locations (the content received by the user remains the same but location and physical space change). For a rapid prototype of this kind of applications, it is important to represent the contents independently of each location.

We have been working on different modelling aspects of mobile applications ([5] and [6]). In particular, we have used the concept of separation of concerns to handle them in an independent way. For instance, having content and location concerns decoupled one another. This experiences that combine location-aware applications with actors' performances are faster to prototype if we use the concept of separation of concerns.

In this paper, we present a model to define location-aware experiences where content aspects are decoupled from location 
ones. To test our model, we create a location-aware application prototype. We describe an in-situ evaluation of this application, which combines location-aware content with actors' performances. We present various results and discussions points of this evaluation.

This paper is organised as follows. In Section 2 we describe some related work. A modelling approach for location-aware experiences is presented in Section 3. In Section 4, we describe a prototype which was created by using our model. An evaluation of this prototype is presented in Section 5. Conclusions and future work are mentioned in Section 6.

\section{RELATED WORK}

In [4], the authors analyse different kinds of Mobile Guides and give the taxonomy of relevant contexts in these kinds of applications, such as the location, that is one of the most useful contexts. The authors describe different location techniques and environment types. In [3], the authors mention the importance of evaluating prototypes in an in-situ way.

An event-driven model is used in [13] to create mobile context aware-applications. The authors build a mobile tourist guide using IVO platform (retrieving locations using GPS), which is tested insitu.

In [10], the authors present a sculptural location-based hypertext model. This model is defined through a characterisation of three patterns (Canyons, Deltas and Plains) that authors have detected in this kind of applications. This model defines the concept of location-query as an attribute of different classes. To test their model, the authors have created GeoYarn, a web service application for Android implemented with this model which provides stories in different locations. Motivated by this idea, we have tested our model in the same way.

Six Mobile Urban Dramas are described in [8]. The authors of this paper present a conceptual framework that includes two specific models (user and environment) to define dramas whose plot is described by means of graphs with branches. The framework detects the user's locations through 2D barcodes, RFID or GPS and, when the user reaches a specific location, he/she receives a multimedia content (audios, images, animations or videos). Each drama is deployed as a mobile application both in iOS and Android platforms.

The authors in [8], [10] and [13] use different models to represent the content and the relevant locations. But in all of them, these concepts are coupled. We use these approaches as an inspiration and as a starting point to define a new model, where the content and location aspects are decoupled.

\section{MODELLING LOCATION-AWARE EXPERIENCES}

In Figure 1, we present a general schema of our model that allows representing location-aware experiences. The abstract class Content represents a general content. For simplicity we have decided not to show each concrete subclass (as Text, Image, Video, etc). We have decoupled the content from the structure that contains it. To do that, we have created the concept of Element. This enables us to reuse a content more than once in different structures (or patterns as mentioned in [10]). The location is defined as an Interface (following the idea of [9] in which a location can be represented, for example, in a symbolic or geometric way). The class PoI acts as a weaving between elements and locations. Note that the same content or position could be used by different PoI.

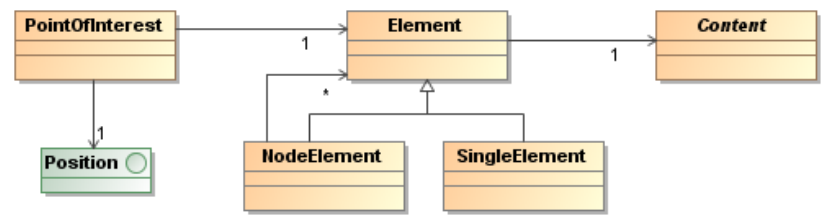

Figure 1. Decoupled contents, structures and locations.

Other relevant classes are required to be able to define the experience itself. To achieve that, we have created the class MobileExperience that contains all the relevant PoI. To show how we achieve the decoupling of some relevant aspects, we point out here the classes SensingManager and SpaceRepresentationManager. These classes are shown in Figure 2. This is a simplified model which aims at presenting the general idea.

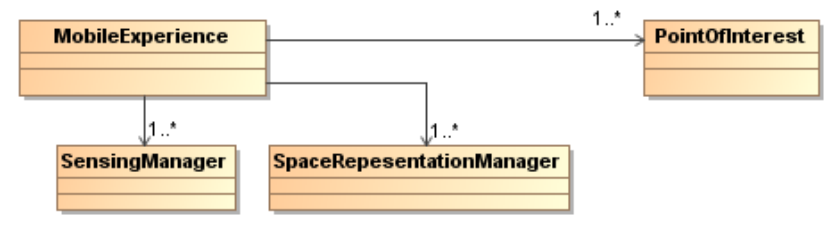
Figure 2. Simplified model to define location-aware
experiences.

\section{OUR PROTOTYPE}

In 2014, we were invited by one of the local TED talks (TEDxDiagonales73) to create a mobile application to be used among the participants. Using the model depicted in Section 3, we decided to create a location-aware application prototype called CaminosAlternativos (Alternative Paths). We defined the indoor space by drawing a plan of the building, including some relevant points such as the elevator and the stairs.

When we first thought about this prototype, we agreed to make a web application and hosted it in a server, so that the participants could access it through the Internet. Later, when we tested the WiFi connection of the building where the TED talk was going to be held, we found out that it was not working properly. Therefore, we decided that the fewer HTTP connections the prototype required, the better performance it would be able to achieve. This is why we decided to use the framework Phonegap [11] to create a hybrid mobile web application for Android. In this way, users would install the mobile application in their phones and the prototype would only have one HTTP connection to send the result generated by each user.

In order to create a more interesting application we thought about combing this application with acting performances in each location. To do so, we contacted Lorena Velazquez, headmaster of the ECAE School of Performing Arts, who helped us to define each acting performance and coordinate the actors' participation. Lorena proposed us doing an experience in which three performances would be defined, each one based on a popular TED talk phrase taken from previous TED events (these phrases were selected by the organisers of the TEDxDiagonales 73 talk). A 
user would see the plays and answer, in one word, what has he/she felt while seeing it. Once the user finishes seeing the three plays, he/she would have to create a phrase trying to use the three words he/she has answered. The user with the most creative phrase would be the winner of a prize at the end of the TEDxDiagonales73 talk.

To achieve this, we visited the building (in which the talk was going to be held) and defined three relevant locations (corresponding to three rooms or scenes), two on the first floor and the other one on the second floor. The way to get around the three locations was without a predetermined order. The first QRcode selected by the user determined the first play he/she would see, which is carried by an actor as shown in Figure 3 (so, there are three actors, one for each scene). Even though this first-scene selection could have been achieved by a technical solution, we decided to do it in this way in order to make a more exciting experience.

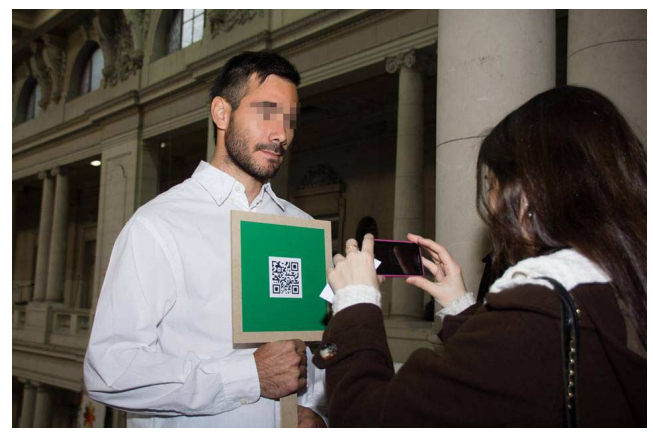

Figure 3. Deciding the first place.

After reading the first QR-code (Figure 3), the application shows a map to the user indicating the path to reach the specific room where one of the performances will take place. The user walks to that room and finds another QR-code, as shown in Figure 4. When the user reads this code a question appears and the user is intended to answer a word regarding to his/her feelings about the performance.

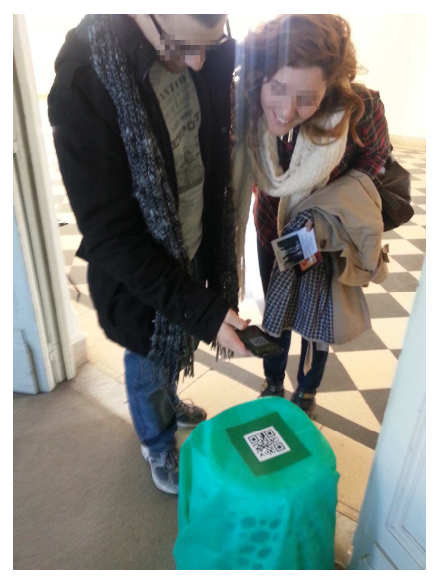

Figure 4. QR-code of a scene.

Once the play is over (all of them last 5 minutes) and the user has answered the question, the application shows the map of the building with the other two scenes that the user still has to see. If the two remaining plays are the ones on the first floor, a map only of this floor is displayed to the user. Otherwise, the user has to choose which floor he/she wishes to go next (see Figure 5).

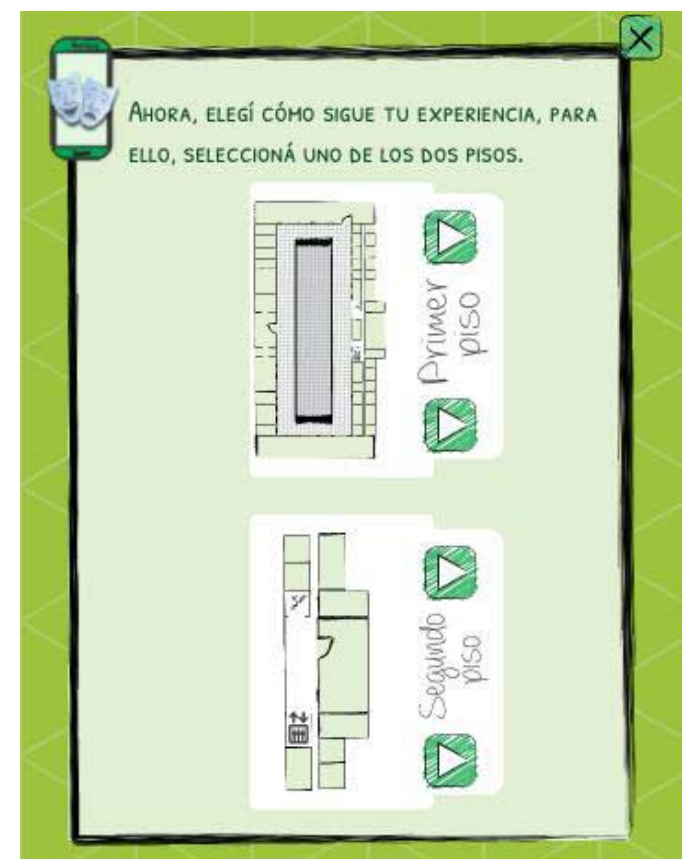

Figure 5. Two options to follow with the experience.

After choosing one of these options (Figure 5), the user receives a map with the path to its destination. If the path involves moving to the other floor, he/she receives the relevant instructions through the maps shown in Figure 6. The map on the left indicates the path to the stairs. Finally, the map on the right assists the user to reach the correct room.

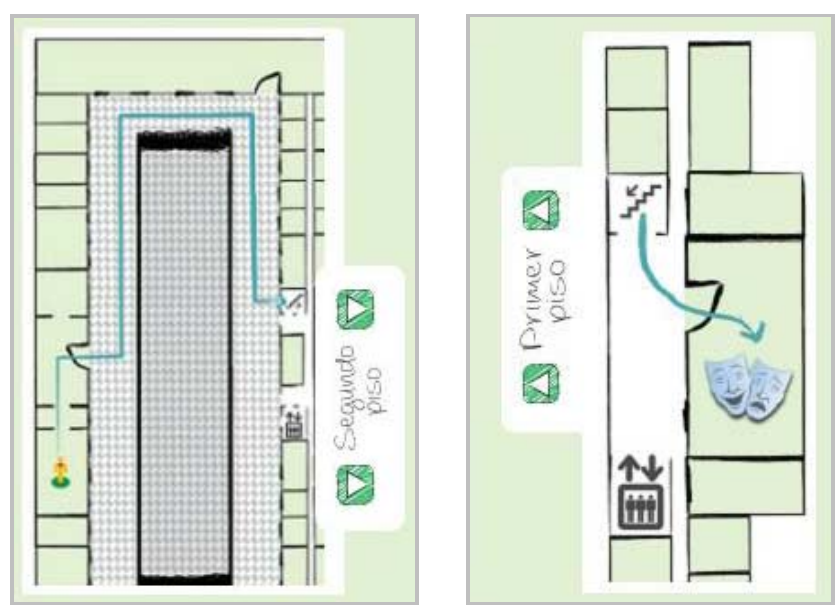

Figure 6. Instruction to move from one floor to the other.

The same process which took place in the first scene is repeated. Since the application was defined with only three scenes, the path to the last scene is automatically provided when the second question is answered. When the user completes the last question, he/she receives a final question to generate a phrase about how the performances have made him/her feel.

Note that the pictures shown in Figure 3 and 4 are taken during the in-situ evaluation with TEDxDiagonales73 participants. 


\section{EVALUATION}

In this section we present the in-situ evaluation of the prototype described in Section 4. The participants of TEDxDiagonales73 that used the application were 34 (ages ranged from 20 to 47). All participants used their own mobile devices to install the prototype.

\subsection{Analysis and Design}

We created a document to describe general characteristics of the prototype and what the installation process in their mobile devices was like. This information was available one day before the TEDxDiagonales 73 talk. In this way, the user had the chance to download it and have it installed by the time of the talk.

We made identification cards with a QR-code and gave them to the users when the experience started. To start running the prototype users were required to read the code of their cards so as to be able to receive the map to go to the place where the three actors were. This identification step was made both to prevent users from running the prototype before the time the evaluation began as well as to create more expectations. Figure 7 shows users reading their identification codes.

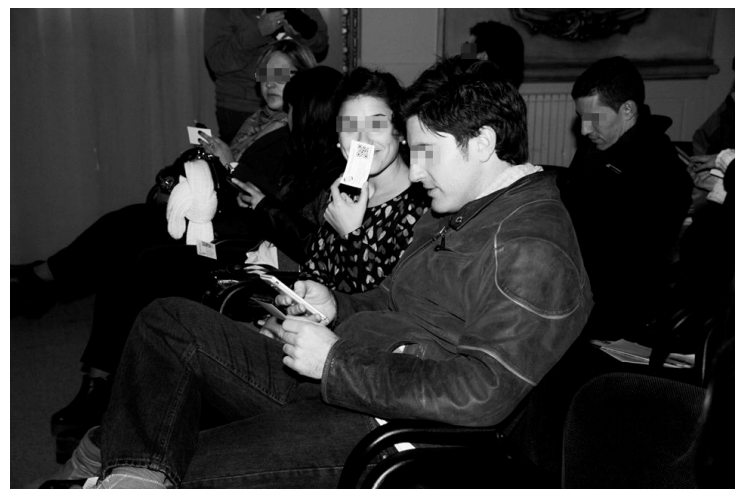

Figure 7. QR-code for identification.

We defined a SUS questionnaire [1] to evaluate the usability of the system. In addition, we defined a general form with two questions:

- Mark the grade of complexity on the use of the application (scores 1 to 5, 1 means low difficulty and 5 high difficulty)

- The assistance provided by the application was enough to move from one place to another (scores 1 to 5,1 means strongly disagree and 5 strongly agree)

These forms were completed by the participants at the end of the experience.

\subsection{Result and Discussions}

The 34 participants completed both forms mentioned in the previous section. The result of the SUS was 76,0 (over 100), which is an acceptable score for a prototype and considering that the indoor space was represented only with and static image that limited the usability of the user.

The result of the general form is described below. The average score for the question "Mark the grade of complexity on the use of the application" was $2,14 \quad(\mathrm{SD}=1,23)$. This means that the complexity to use the prototype was low. The other question "The assistance provided by the application was enough to move from place to another" had an average score of $3,79(\mathrm{SD}=1,27)$. According with these values the assistance is more open to discussion because it was useful to most of the users but for others it was not. This point requires further improvement in future prototypes.

In this section we discuss some interesting points. To prevent all the users from going to the same play, each actor (of the three initial actors shown in Figure 3) counted the number of participants that were reading his QR-code. When this number reached the number of 12 users, the actor hid the code so that no other user could read it. This tactic was thought to balance the number of users in each room (or scene) preventing each room from getting overcrowded.

Another issue came up at the time of choosing the second play to see. Participants that were on the first floor (where two performances were being held), preferred their next choice to be the one of the first floor. In this situation, there were fewer people on the second floor than on the first one, where most participants were.

All three performances required a clockwork synchronisation to start simultaneously. To solve this, in each scene there were assistants who saw to this and decided when to start each performance, so as to do it all at the same time. The assistants' mission was also guaranteeing that there were no participants in the corridor or stairs.

The QR-code identifications were general, so there was no need to give any personal information. This was an advantage due to the fact that being anonymous increased the chances that users would answer the questions.

Both the content and the structure can be reused as the application was built following the model presented in the Section 3 . To reuse them, it will only be necessary to define a new physical space to be able to test this prototype.

\section{CONCLUSION AND FUTURE WORK}

In this paper, we have presented a model to location-aware experiences. We described the main classes and focused on providing decoupled aspects of these kinds of applications, such as contents, structure and locations.

We have described a novel prototype, which was combined with actors' performances. We have presented and analysed the result of the evaluation in-situ. The discussion points mentioned in this paper are part of the learning process in evaluation in-situ. This type of evaluations (with real end-user people) are the best way to understand how these kind of applications behave.

We have presented the evaluation of this application and then we discussed some interesting aspects that were learnt as part of the in-situ evaluation.

We are working to enrich our model and create more complex applications. One of the aspects to improve is the map the application provides. In indoor spaces it is essential to be precise in the assistance to ensure that the user arrives at his/her destination as easily and straightforward as possible. Moreover, based on this model, we are currently developing a tool that enables end-users to create these location-aware experiences. 
As a future work, we will evaluate this model with other prototypes to consider other relevant aspects. For example, the collaboration between users to create contents.

\section{ACKNOWLEDGMENTS}

We extend our thanks to Lorena Velazquez for creating and preparing each acting performance, and to the actors of ECAE (Espacio Creativo de las Artes Escénicas). The authors thank to Agustina Zimbello and Leandro Vilas for their collaboration on the organisation of this experience.

\section{REFERENCES}

[1] Brooke, J. 1996. SUS-A quick and dirty usability scale. Usability evaluation in industry 189,194, 4-7.

[2] Consolvo, S., Harrison, B., Smith, I., Chen, M. Y., Everitt, K., Froehlich, J., and Landay, J. A. 2007. Conducting in situ evaluations for and with ubiquitous computing technologies. International Journal of Human-Computer Interaction 22, 12, (Dec. 2007), 103-118.

[3] Crabtree, A., Chamberlain, A., Davies, M., Glover, K., Reeves, S., Rodden, T., and Jones, M. 2013. Doing innovation in the wild. In Proceedings of the Biannual Conference of the Italian Chapter of SIGCHI (Trento, Italy, September16-19, 2013). CHItaly '13. ACM, New York, NY, Article No. 25. DOI=

http://dl.acm.org/citation.cfm?doid=2499149.2499150

[4] Emmanouilidis, C., Koutsiamanis, R. A., and Tasidou, A. 2013. Mobile guides: Taxonomy of architectures, context awareness, technologies and applications. Journal of Network and Computer Applications 36, 1, (Jan. 2013), 103125 .

[5] Fortier, A., Challiol, C., Fernández, J. L., Robles, S., Rossi, G., and Gordillo, S. 2014. Exploiting personal web servers for mobile context-aware applications. The Knowledge Engineering Review 29, 2, (Mar. 2014), 134-153. DOI=http://dx.doi.org/10.1017/S0269888914000022

[6] Fortier, A., Rossi, G., Gordillo, S. E., and Challiol, C. 2010. Dealing with variability in context-aware mobile software. Journal of Systems and Software 83, 6, (June 2010) 915-936. $\mathrm{DOI}=$

http://www.sciencedirect.com/science/article/pii/S01641212 09002830

[7] Hansen, F. A., Kortbek, K. J., and Grønbæk, K. 2008. Mobile Urban Drama-Setting the Stage with Location Based Technologies. In Proceeding Proceedings of the 1st Joint International Conference on Interactive Digital Storytelling: Interactive Storytelling (Erfurt, Germany, November 26-29, 2008). ICIDS '08. Springer-Verlag Berlin, Heidelberg, 2031.
[8] Hansen, F. A., Kortbek, K. J., and Grønbæk, K. 2012. Mobile urban drama: interactive storytelling in real world environments. New Review of Hypermedia and Multimedia 18, 1-2, (Mar.-June 2012) 63-89. DOI= http://www.tandfonline.com/doi/abs/10.1080/13614568.2012 .617842

[9] Leonhardt, U. 1998. Supporting location-awareness in open distributed systems. Doctoral dissertation, Imperial College.

[10] Millard, D. E., Hargood, C., Jewell, M. O., and Weal, M. J. 2013. Canyons, deltas and plains: towards a unified sculptural model of location-based hypertext. In Proceedings of the 24th ACM Conference on Hypertext and Social Media (Paris, France, May 01-03, 2013). Hypertext 2013. ACM, New York, NY, 109-118. DOI= http://dl.acm.org/citation.cfm?doid=2481492.2481504

[11] Phonegap Home Page: http://phonegap.com/

[12] Pittarello, F. 2011. Designing a context-aware architecture for emotionally engaging mobile storytelling. In Proceeding Proceedings of the 13th IFIP TC 13 international conference on Human-computer interaction (Lisbon, Portugal, September 5-9, 2011). INTERACT '11. Springer-Verlag Berlin, Heidelberg, 144-151.

[13] Realinho, V., Romão, T., Birra, F., and Dias, A. E. 2011.Building mobile context-aware applications for leisure and entertainment. In Proceedings of the 8th International Conference on Advances in Computer Entertainment Technology (Lisbon, Portugal, 8-11 November, 2011). ACE 2006. ACM, New York, NY, Article No. 29.

DOI=http://dl.acm.org/citation.cfm?doid=2071423.2071459

[14] Rogers, Y., Connelly, K., Tedesco, L., Hazlewood, W., Kurtz, A., Hall, R. E., and Toscos, T. 2007. Why it's worth the hassle: The value of in-situ studies when designing ubicomp. In Proceeding of the 9th international conference on Ubiquitous Computing (Innsbruck, Austria, September 16-19, 2007). UbiComp 2007. Springer Berlin Heidelberg, 336-353.

[15] Santos, P., Hernández-Leo, D., and Blat, J. 2014. To be or not to be in situ outdoors, and other implications for design and implementation, in geolocated mobile learning. Pervasive and Mobile Computing 14, (October, 2014), 1730.

[16] Weal, M. J., Hornecker, E., Cruickshank, D. G., Michaelides, D. T., Millard, D. E., Halloran, J., and Fitzpatrick, G. 2006. Requirements for in-situ authoring of location based experiences. In Proceedings of the 8th conference on Human-computer interaction with mobile devices and services (Helsinki, Finland, September 12-15, 2006). Mobile HCI 2006. ACM, New York, NY, 121-128. DOI= http://dl.acm.org/citation.cfm?doid=1152215.1152241

\footnotetext{
\# Another affiliation for Cecilia Challiol: CONICET, Argentina.

${ }^{\S}$ Another affiliation for Silvia E. Gordillo: CIC, Buenos Aires, Argentina.
} 